\title{
ON THE DERIVATIVE AND MAXIMUM MODULUS OF A POLYNOMIAL
}

\author{
K. K. DEWAN, N. K. GOVIL, ABDULLAH MIR, AND M. S. PUKHTA
}

Received 2 October 2004; Accepted 11 October 2004

If $p(z)=\sum_{v=0}^{n} a_{v} z^{v}$ is a polynomial of degree $n$, having all its zeros in $|z| \leq 1$, then it was proved by Turán that $\left|p^{\prime}(z)\right| \geq(n / 2) \max _{|z|=1}|p(z)|$. This result of Turán was generalized by Govil, who proved that if $p(z)$ has all its zeros in $|z| \leq K, K \geq 1$, then $\max _{|z|=1}\left|p^{\prime}(z)\right| \geq$ $\left(n /\left(1+K^{n}\right)\right) \max _{|z|=1}|p(z)|, K \geq 1$. In this paper, we sharpen this, and some other related results.

Copyright (c) 2006 K. K. Dewan et al. This is an open access article distributed under the Creative Commons Attribution License, which permits unrestricted use, distribution, and reproduction in any medium, provided the original work is properly cited.

\section{Introduction and statement of results}

If $p(z)=\sum_{v=0}^{n} a_{v} z^{v}$ is a polynomial of degree $n$, then it is well known that

$$
\max _{|z|=1}\left|p^{\prime}(z)\right| \leq n \max _{|z|=1}|p(z)| .
$$

The above inequality, which is an immediate consequence of Bernstein's inequality on the derivative of a trigonometric polynomial, is best possible with equality holding for the polynomial $p(z)=\lambda z^{n}, \lambda$ being a complex number.

If we restrict ourselves to the class of polynomials having no zeros in $|z|<1$, then the above inequality can be sharpened. In fact Erdös conjectured and later Lax [7] proved that if $p(z) \neq 0$ in $|z|<1$, then

$$
\max _{|z|=1}\left|p^{\prime}(z)\right| \leq \frac{n}{2} \max _{|z|=1}|p(z)|
$$

If the polynomial $p(z)$ of degree $n$ has all its zeros in $|z| \leq 1$, then it was proved by Turán [9], that

$$
\max _{|z|=1}\left|p^{\prime}(z)\right| \geq \frac{n}{2} \max _{|z|=1}|p(z)|
$$

Hindawi Publishing Corporation Journal of Inequalities and Applications Volume 2006, Article ID 54816, Pages 1-6 DOI 10.1155/JIA/2006/54816 
2 On derivative and maximum modulus of a polynomial

The inequalities (1.2) and (1.3) are also best possible, and become equality for polynomials which have all its zeros on $|z|=1$.

The above inequality (1.3) of Turán [9] was generalized by Govil [3], who proved that if $p(z)$ is a polynomial of degree $n$ having all its zeros in $|z| \leq K$, then

$$
\begin{aligned}
& \max _{|z|=1}\left|p^{\prime}(z)\right| \geq \frac{n}{1+K} \max _{|z|=1}|p(z)|, \quad \text { if } K \leq 1, \\
& \max _{|z|=1}\left|p^{\prime}(z)\right| \geq \frac{n}{1+K^{n}} \max _{|z|=1}|p(z)|, \quad \text { if } K \geq 1 .
\end{aligned}
$$

Both the above inequalities are best possible, with equality in (1.4) holding for $p(z)=$ $(z+K)^{n}$, while in (1.5) the equality holds for the polynomial $p(z)=z^{n}+K^{n}$. The inequality (1.4) was also proved by Malik [8].

The inequality (1.5) was later sharpened by Govil [4, page 67], who proved the following theorem.

Theorem 1.1. If $p(z)=\sum_{v=0}^{n} a_{v} z^{v}, a_{n} \neq 0$, is a polynomial of degree $n$ having all its zeros in $|z| \leq K, K \geq 1$, then

$$
\begin{aligned}
\max _{|z|=1}\left|p^{\prime}(z)\right| \geq & \frac{n}{1+K^{n}} \max _{|z|=1}|p(z)| \\
& +\frac{n\left|a_{n-1}\right|}{K\left(1+K^{n}\right)}\left(\frac{K^{n}-1}{n}-\frac{K^{n-2}-1}{n-2}\right)+\left|a_{1}\right|\left(1-\frac{1}{K^{2}}\right)
\end{aligned}
$$

if $n>2$, and

$$
\max _{|z|=1}\left|p^{\prime}(z)\right| \geq \frac{n}{1+K^{n}} \max _{|z|=1}|p(z)|+\frac{K^{n}-1}{K^{n}+1}\left|a_{1}\right|
$$

if $n=2$.

The above inequalities are best possible and are attained for the polynomial $p(z)=z^{n}+$ $K^{n}$.

In this paper, we prove the following refinement of Theorem 1.1, which in turn gives the refinements of inequalities (1.3), and (1.5).

TheOREM 1.2. If $p(z)=\sum_{v=0}^{n} a_{v} z^{v}, a_{n} \neq 0$, is a polynomial of degree $n$ having all its zeros in $|z| \leq K, K \geq 1$, then

$$
\begin{aligned}
\max _{|z|=1}\left|p^{\prime}(z)\right| \geq & \frac{n}{1+K^{n}}\left\{\max _{|z|=1}|p(z)|+\min _{|z|=K}|p(z)|\right\}+\left|a_{1}\right|\left(1-\frac{1}{K^{2}}\right) \\
& +\frac{n\left|a_{n-1}\right|}{K\left(1+K^{n}\right)}\left(\frac{K^{n}-1}{n}-\frac{K^{n-2}-1}{n-2}\right)
\end{aligned}
$$


K. K. Dewan et al. 3

if $n>2$, and

$$
\max _{|z|=1}\left|p^{\prime}(z)\right| \geq \frac{n}{1+K^{n}}\left\{\max _{|z|=1}|p(z)|+\min _{|z|=K}|p(z)|\right\}+\frac{K^{n}-1}{K^{n}+1}\left|a_{1}\right|
$$

if $n=2$.

Both the above inequalities are best possible and are attained for the polynomial $p(z)=$ $z^{n}+K^{n}$.

If we take $K=1$ in the above theorem, we get the following result, which was proved by Aziz and Dawood [1].

Corollary 1.3. If $p(z)=\sum_{v=0}^{n} a_{v} z^{v}, a_{n} \neq 0$, is a polynomial of degree $n$ having all its zeros in $|z| \leq 1$, then

$$
\max _{|z|=1}\left|p^{\prime}(z)\right| \geq \frac{n}{2}\left\{\max _{|z|=1}|p(z)|+\min _{|z|=1}|p(z)|\right\}
$$

\section{Lemmas}

We will need the following lemmas.

Lemma 2.1. If $p(z)$ is a polynomial of degree $n$, having all its zeros in $|z| \leq 1$, then

$$
\max _{|z|=1}\left|p^{\prime}(z)\right| \geq \frac{n}{2}\left\{\max _{|z|=1}|p(z)|+\min _{|z|=1}|p(z)|\right\}
$$

The result is best possible and the equality holds for the polynomial $p(z)=(z+1)^{n}$.

The above result is due to Aziz and Dawood [1] (also see Govil [5, Theorem 2, inequality (1.7)]).

Lemma 2.2. If $p(z)=\sum_{v=0}^{n} a_{v} z^{v}$ is a polynomial of degree $n$, having no zeros on $|z|<1$, then for $R \geq 1$,

$$
\begin{aligned}
\max _{|z|=R \geq 1}|p(z)| \leq & \left(\frac{R^{n}+1}{2}\right) \max _{|z|=1}|p(z)|-\left(\frac{R^{n}-1}{2}\right) \min _{|z|=1}|p(z)| \\
& -\left|a_{1}\right|\left(\frac{R^{n}-1}{n}-\frac{R^{n-2}-1}{n-2}\right), \quad \text { if } n>2, \\
\max _{|z|=R \geq 1}|p(z)| \leq & \left(\frac{R^{n}+1}{2}\right) \max _{|z|=1}|p(z)|-\left(\frac{R^{n}-1}{2}\right) \min _{|z|=1}|p(z)| \\
& -\left|a_{1}\right| \frac{(R-1)^{n}}{2}, \quad \text { if } n=2 .
\end{aligned}
$$

The above result is a special case, with $s=1$ and $K=1$, of a result due to Govil [6, page 625]. 
4 On derivative and maximum modulus of a polynomial

Lemma 2.3. If $p(z)=\sum_{v=0}^{n} a_{v} z^{v}$ is a polynomial of degree $n, n \geq 1$, then for all $R \geq 1$,

$$
\begin{gathered}
\max _{|z|=R}|p(z)| \leq R^{n} \max _{|z|=1}|p(z)|-\left(R^{n}-R^{n-2}\right)|p(0)|, \quad \text { if } n \geq 2, \\
\max _{|z|=R}|p(z)| \leq R \max _{|z|=1}|p(z)|-(R-1)|p(0)|, \quad \text { if } n=1 .
\end{gathered}
$$

The inequality (2.4) is due to Frappier et al. [2, Theorem 2], while (2.5) follows trivially.

\section{Proof of the theorem}

We first consider the case when $p(z)$ is degree $n>2$. Since $p(z)$ has all its zeros in $|z| \leq K$, $K \geq 1$, the polynomial $P(z)=p(K z)$ is of degree $n$, and has all its zeros in $|z| \leq 1$. Hence if we apply Lemma 2.1 to the polynomial $P(z)$, we will get

$$
\max _{|z|=1}\left|P^{\prime}(z)\right| \geq \frac{n}{2}\left\{\max _{|z|=1}|P(z)|+\min _{|z|=1}|P(z)|\right\}
$$

which is equivalent to

$$
K \max _{|z|=K}\left|p^{\prime}(z)\right| \geq \frac{n}{2}\left\{\max _{|z|=K}|p(z)|+\min _{|z|=K}|p(z)|\right\} .
$$

The polynomial $p(z)$ is of degree $n>2$, and so the polynomial $p^{\prime}(z)$ is of degree $n-$ 1 , where $n-1 \geq 2$, and hence applying Lemma 2.3 to the polynomial $p^{\prime}(z)$, we get for $K \geq 1$,

$$
\max _{|z|=K}\left|p^{\prime}(z)\right| \leq K^{n-1} \max _{|z|=1}\left|p^{\prime}(z)\right|-\left(K^{n-1}-K^{n-3}\right)\left|a_{1}\right| .
$$

Combining (3.2) and (3.3), we get for $K \geq 1$,

$$
K^{n-1} \max _{|z|=1}\left|p^{\prime}(z)\right|-\left(K^{n-1}-K^{n-3}\right)\left|a_{1}\right| \geq \frac{n}{2 K}\left\{\max _{|z|=K}|p(z)|+\min _{|z|=K}|p(z)|\right\},
$$

which is equivalent to

$$
K^{n} \max _{|z|=1}\left|p^{\prime}(z)\right|-\left(K^{n}-K^{n-2}\right)\left|a_{1}\right| \geq \frac{n}{2}\left\{\max _{|z|=K}|p(z)|+\min _{|z|=K}|p(z)|\right\} .
$$

Since the polynomial $p(z)$ has all its zeros in $|z| \leq K, K \geq 1$, the polynomial $q(z)=$ $z^{n} p(1 / z)$ has no zeros in $|z|<1 / K$, hence the polynomial $q(z / K)$ is of degree $n>2$, and has no zeros in $|z|<1$. Therefore, on applying Lemma 2.2 to the polynomial $q(z / K)$, we get

$$
\begin{aligned}
\max _{|z|=K \geq 1}|q(z / K)| \leq & \frac{K^{n}+1}{2} \max _{|z|=1}|q(z / K)|-\frac{K^{n}-1}{2} \min _{|z|=1}|q(z / K)| \\
& -\frac{\left|a_{n-1}\right|}{K}\left(\frac{K^{n}-1}{n}-\frac{K^{n-2}-1}{n-2}\right)
\end{aligned}
$$


which is equivalent to

$$
\begin{aligned}
\max _{|z|=1}|p(z)| \leq & \frac{K^{n}+1}{2 K^{n}} \max _{|z|=K}|p(z)|-\frac{K^{n}-1}{2 K^{n}} \min _{|z|=K}|p(z)| \\
& -\frac{\left|a_{n-1}\right|}{K}\left(\frac{K^{n}-1}{n}-\frac{K^{n-2}-1}{n-2}\right) .
\end{aligned}
$$

The above inequality easily gives

$$
\begin{aligned}
\max _{|z|=K}|p(z)| \geq & \frac{2 K^{n}}{K^{n}+1} \max _{|z|=1}|p(z)|+\frac{K^{n}-1}{K^{n}+1} \min _{|z|=K}|p(z)| \\
& +\frac{2 K^{n-1}}{1+K^{n}}\left|a_{n-1}\right|\left(\frac{K^{n}-1}{n}-\frac{K^{n-2}-1}{n-2}\right),
\end{aligned}
$$

and this when combined with (3.5) gives

$$
\begin{aligned}
& \frac{2 K^{n}}{n} \max _{|z|=1}\left|p^{\prime}(z)\right|-\frac{2\left(K^{n}-K^{n-2}\right)}{n}\left|a_{1}\right|-\min _{|z|=K}|p(z)| \\
& \quad \geq \frac{2 K^{n}}{K^{n}+1} \max _{|z|=1}|p(z)|+\frac{K^{n}-1}{K^{n}+1} \min _{|z|=K}|p(z)|+\frac{2 K^{n-1}}{1+K^{n}}\left|a_{n-1}\right|\left(\frac{K^{n}-1}{n}-\frac{K^{n-2}-1}{n-2}\right) .
\end{aligned}
$$

The above inequality (3.9) is clearly equivalent to

$$
\begin{aligned}
\max _{|z|=1}\left|p^{\prime}(z)\right| \geq & \left|a_{1}\right|\left(1-\frac{1}{K^{2}}\right)+\frac{n}{K^{n}+1}\left(\max _{|z|=1}|p(z)|+\min _{|z|=K}|p(z)|\right) \\
& +\frac{n\left|a_{n-1}\right|}{K\left(1+K^{n}\right)}\left(\frac{K^{n}-1}{n}-\frac{K^{n-2}-1}{n-2}\right),
\end{aligned}
$$

which is inequality (1.8), and thus our theorem, in the case $n>2$, is proved.

The proof of the theorem in the case $n=2$ follows on the same lines as above except that instead of inequalities (2.2) and (2.4), we use inequalities (2.3) and (2.5), respectively.

\section{References}

[1] A. Aziz and Q. M. Dawood, Inequalities for a polynomial and its derivative, Journal of Approximation Theory 54 (1988), no. 3, 306-313.

[2] C. Frappier, Q. I. Rahman, and St. Ruscheweyh, New inequalities for polynomials, Transactions of the American Mathematical Society 288 (1985), no. 1, 69-99.

[3] N. K. Govil, On the derivative of a polynomial, Proceedings of the American Mathematical Society 41 (1973), 543-546.

[4] _ Inequalities for the derivative of a polynomial, Journal of Approximation Theory 63 (1990), no. 1, 65-71.

[5] _ Some inequalities for derivatives of polynomials, Journal of Approximation Theory 66 (1991), no. 1, 29-35.

[6] - On growth of polynomials, Journal of Inequalities and Applications 7 (2002), no. 5, 623-631. 


\section{On derivative and maximum modulus of a polynomial}

[7] P. D. Lax, Proof of a conjecture of P. Erdös on the derivative of a polynomial, American Mathematical Society. Bulletin 50 (1944), 509-513.

[8] M. A. Malik, On the derivative of a polynomial, Journal of the London Mathematical Society. Second Series 1 (1969), 57-60.

[9] P. Turán, Über die Ableitung von Polynomen, Compositio Mathematica 7 (1939), 89-95 (German).

K. K. Dewan: Department of Mathematics, Jamia Millia Islamia, New Delhi 110025, India E-mail address: kkdewan123@yahoo.co.in

N. K. Govil: Department of Mathematics, Auburn University, Auburn, AL 36849-5310, USA E-mail address: govilnk@mail.auburn.edu

Abdullah Mir: Department of Mathematics, Jamia Millia Islamia, New Delhi 110025, India

M. S. Pukhta: Department of Mathematics, Jamia Millia Islamia, New Delhi 110025, India 